\title{
In vitro degradation of polydioxanone (PDO) lifting threads in hyaluronic acid (HA)
}

Dubraska V Suárez-Vega, MSc ${ }^{1}$; Gladys J Velazco de Maldonado, PhD²; Reynaldo Ortiz, $\mathrm{PhD}^{3}$; Victor Garcia-Guevara, $\mathrm{MD}^{4}$; Blanca Miller-Kobisher, MD, MMed, MScNut ${ }^{5}$.

1. Magister in Medicine. Doctoral Student. Professor at the University of Los Andes (ULA), Mérida-Venezuela. Faculty of Dentistry. Department of Investigation. Dental Research Center.

2. Doctor of Medicine, PhD. Professor at the University of Los Andes (ULA), Mérida-Venezuela. Faculty of Dentistry. Department of Restorative Dentistry. Center for Dental Research.

3. PhD in electrochemistry, PhD. Professor at the University of the Andes (ULA), MéridaVenezuela Faculty of Sciences. Chemistry Department. Electrochemistry Laboratory.

4. Master's degree in Aesthetic Medicine. Academic Director of the Aesthetic Medicine Studies Center Foundation (FUCEME), Caracas, Venezuela.

5. Master's Degree in Medical Education and Master's Degree in Clinical Nutrition. Academic Director of the Pan-American Institute of Scientific Professionals (IPPC) Mexico

Study performed at Faculty of Dentistry. Department of Investigation. Dental Research Center Financial support: None.

Conflict of interest: None.

Correspondence address: Dubraska Suárez. Faculty of Dentistry, 23rd Street between avenues 2 and 3, Research Department, University of Los Andes (ULA), Mérida-Venezuela. Phone: + 580274240237. E-mail: dubraskasuarez.ula@gmail.com

Submitted: october 22, 2018; accepted after revision, April, 19, 2019.

\section{ABSTRACT}

Background: Recently, some clinicians have proposed implanting PDO threads imbibed in hyaluronic acid (HA). However, this is controversial since PDO sutures are hydrophilic and the presence of HA could increase the rate of hydrolysis. Aim: To demonstrate the degradation of PDO lifting threads in HA through ultramicroscopy. Methods: Three, one $\mathrm{cm}$ long, segments of 23G PDO threads, where immersed in $1.5 \mathrm{ml}$ non-crosslinked hyaluronic acid in previously labeled, sterile microcentrifuge tubes. These were observed by ultramicroscopy at $4 x$ and $10 x$ after 24, 48 and 72 hours. Results: Microphotographs taken after 24 hours already show structural changes in the fibers, presenting an increase in interlaminar spaces and dilution of violet pigmentation. At 48 hours, degradation continues. PDO hygroscopy is observed as aqueous content between the peripheral layers and the central core of the thread. Some fibers show breakage, and there is an increase in interlaminar and interfibrillar spaces. At 72 hours, as the pigment is released, larger empty spaces are observed in the central column of the 
thread, and there is disorganization of the peripheral fibrils with fraying all along the fiber. Conclusions: Hyaluronic acid induces rapid biodegradation of the PDO thread by hydrolysis beginning 24 hours after contact of the thread with the biomaterial. We hypothesize that non-crosslinked hyaluronic acid is a powerful catalyzing agent for hydrolytic degradation of the PDO thread, since this thread is highly hydrophilic. Thus, we suggest that clinically embedding PDO threads with HA will only accelerate biodegradation of the suture.

Keywords: Lifting threads, polydioxanone, hyaluronic acid, biodegradation, hydrolysis.

\section{INTRODUCTION}

The use of lifting threads for minimally invasive facial rejuvenation has traversed many phases since from the pioneering proposal of Russian Doctor Sulamanidze et $\mathrm{al}^{1}$, using polypropylene, non-absorbable sutures (Russian Threads) to the recent tendencies that prefer implanting reabsorbable sutures ${ }^{2}$. Among the many variations of threads that have emerged, we find polydioxanone or PDO threads that are designed from this reabsorbable polymer due to its biocompatibility. This technique became popular with the name of Japanese Lifting, since these threads are manufactured in Japan or South Korea $\left(\text { Miracu }^{\circledR}, \text { MesoTraX }^{\circledR}, \text { Mesolift }^{\circledR}, \text { V-lift }^{\circledR}\right)^{3}$.

Polydioxanone is a violet or colorless homopolymer of paradioxanone(1,4dioxane-2-one), is an biodegradable suture, highly flexible and reabsorbable. Structurally, polydioxanone is a polyester-ether differentiated from polyglycolytic acid by an ester group substituted by an ether group, making it much more flexible 4 .

Furthermore, by reducing the concentration of ester groups, polydioxanone is degraded more slowly, conserving its mechanical resistance for longer time periods. It remains in the tissues for a number of weeks (up to 240 days) ${ }^{5}$. Thus, it has been preferentially used in cardiovascular, ophthalmic and pediatric surgery ${ }^{6,7,8}$.

Synthetic suture materials, such as PDO, are absorbed after a process of hydrolysis through the action of intercellular water. The amount of absorbed water determines the degree of hydrolysis of the polymer. In those sutures, white blood cells (mainly macrophages) phagocytize the chains that are initially broken into shorter fragments. The PDO polymer is degraded into low molecular weight, non-toxic residues 
that are eliminated from the organism through normal metabolic pathways: urine, feces, and respiration (exhaling $\mathrm{CO} 2)^{4}$.

PDO thread face lifts have been a successful therapy indicated for reverting sagging, lipomatosis, rhytidosis, and deep folds when the degree of aging does not merit surgical treatment. After the thread is inserted, the organism recognizes it and stabilizes it through mechanisms involving lymphocyte infiltration, collagen deposition and a fibrotic reaction surrounding the biomaterial, mediated by myofibroblasts ${ }^{2,9,10}$. Thus, the lifting effect on sagging tissues is due to fibrotic paths organized during the permanence of the thread and the residual path once the suture is reabsorbed. The PDO begins to have an effect on the skin after two weeks, when collagen production becomes intense $\mathrm{e}^{-11}$.

In spite of important advances in Esthetic and Regenerative Medicine, some clinicians have arbitrarily tried to popularize a clinical protocol, with no foundation in evidence-based medicine, which consists of associating PDO threads to hyaluronic acid, under the supposition that this association will potentiate the lift, based on the skin turgor observed when applying minimally cross-linked HA fillers in wrinkles and deep folds. However, this proposal is considered controversial and even inadmissible to the authors of this article, since polydioxanone is highly hydrophilic and thus, in the presence of $\mathrm{HA}$, hydrolysis could occur very quickly, risking loss of the results pursued with the implantation of the threads.

Thus, in order to elucidate how coherent the conjugation of hyaluronic acid with PDO threads really is, we proposed this investigation that aims to demonstrate the degradation of polydioxanone lifting threads in hyaluronic acid through ultramicroscopy.

\section{METHODS}

We analyzed a monofilament of polydioxanone Brand JBP V-Lift Premium ${ }^{\circledR}$ (JBP KOREA CO.,LTD.), with a $23 \mathrm{G}$ (4-0) diameter, $150 \mathrm{~mm}$ in length. The suture was sterilized and vacuum packed with no degree of humidity. The thread was extracted from the guiding cannula on a sterile field, and using a metallic millimetric ruler, measurements were taken to cut three, one $\mathrm{cm}$ long, segments with sterile surgical scissors. 
To carry out in vitro degradation studies, we used non-crosslinked hyaluronic acid as hydrolysis media, HYALURONIC ACID INNO-TDS ${ }^{\circledR}$ (formula 1567@ Laboratories INNOAESTHETICS, S.L. Barcelona-Spain), which showed an initial pH of 6.5 to 7.0. Each segment of PDO thread was separately submerged in $1 \mathrm{ml}$ non-crosslinked hyaluronic acid in sterile $1.5 \mathrm{ml}$ polypropylene microcentrifuge tubes, labeled for sample identification and divided into three groups, depending on immersion times. Once the tubes were closed, they were incubated at $37^{\circ} \mathrm{C}$.

After the evaluation period (24, 48 and 72 hours after immersion), the PDO suture segments were taken from the tube with sterile tweezers, dried over a sterile field at room temperature for 15 minutes, mounted on a microscope slide, and observed ultramicroscopically under a Nikon ${ }^{\circledR}$ reflected light optical microscope at $4 x$ and 10x. We carried out a microphotographic scan of each segment obtaining at least 12 images three for each of the groups.

\section{RESULTS}

The findings illustrate the degradation of PDO threads as a function of time, demonstrating that the surface of the sample shows changes associated with hydrolytic degradation of the system after 24,48 and 72 hours of contact with hyaluronic acid.

In the first place, microphotographs taken after 24 hours already show structural changes in the fibers with an increase in interlaminar spaces. This is visualized more clearly in the central column of the thread where there are areas of discontinuous dilution of the violet pigment (Figure 1).

a

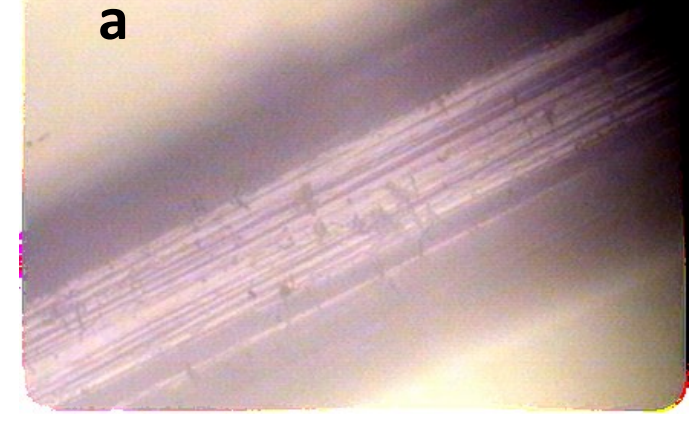

b

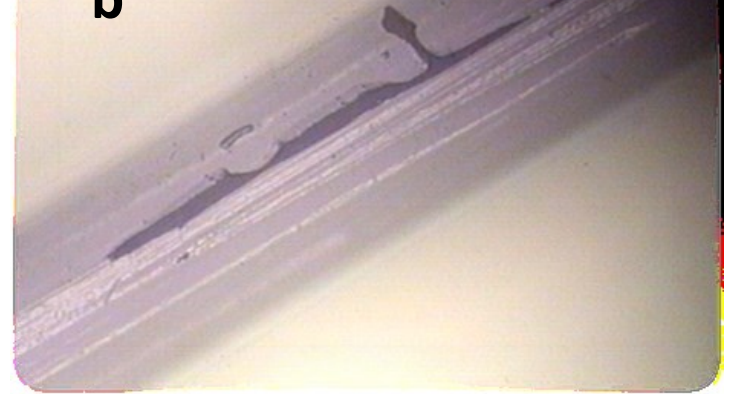

Figure 1 - PDO thread after 24 hours of immersion in HA.

a. Left, reflected light microphotography of the thread at 10xwhich shows the widened interlaminar and interfibrillar spaces in the central column of the thread as vertical, parallel whitish bands corresponding to the dissolution of the amorphous phase of the polymer. b. Right, reflected light microphotograph of the thread at $4 x$ which shows traces of pigment being expelled toward the 
In vitro degradation of polydioxanone (PDO) lifting threads in hyaluronic acid (HA)

Suarez-Vega DV, et al

periphery of the thread on a central background of interlaminar and interfibrillar empty spaces corresponding to zones of fiber hydrolysis.

Assessment of the next segment, corresponding to 48 hours of immersion, suggests that degradation continues by hygroscopy of PDO, observing serpentine swelling in the periphery of the thread and an increase in interlaminar spaces (Figure 2 $a$ and $b)$.

An undulating heterogeneous zone that may correspond to the water absorption phenomenon at the periphery. Additionally, we can visualize retention of the aqueous content between the peripheral layers and the central core of the thread, indicating a hygroscopic phenomenon experienced by the fiber in the presence of HA, which seems to show a pattern of hydrolysis by layers or interlaminar in this second stage (Figure $2 \mathrm{c}$ and d). 

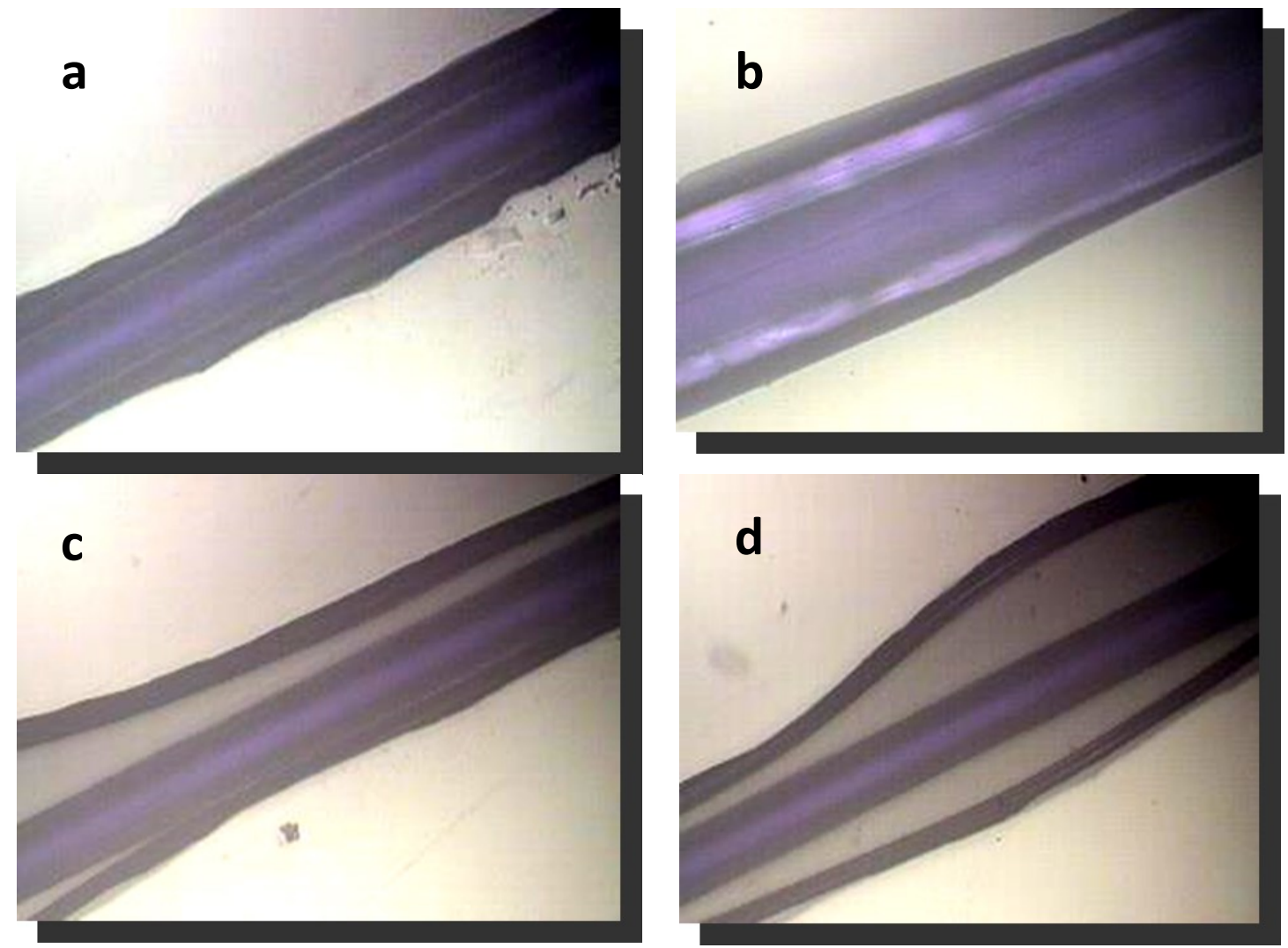

Figure 2 - PDO thread after 48 hours of immersion in HA.

a. microphotography of the thread at $4 x$. Note the central column of the thread showing a clear background surrounded by parallel layers in dark and light up to the periphery of the thread. b. Area at10x. Note the increase in bright interfibrillar spaces seen as vertical parallel whitish bands corresponding to the dissolution of the periphery of the thread. $C$ and $d$. the thread at $4 x$. We observe retention of aqueous content between the peripheral layers and the central core of the thread.

Moreover, at 48 hours there is evidence of the marked hydrolytic attack in the amorphous zones and the process continues with degradation by layers in a linear pattern (Figure 3a). This model suggests that the cracks between the layers and between the interfibrillar zones correspond to small empty spaces formed as consequence of the hydrolytic degradation of the amorphous zone, which produces breakage of the bonds in the aliphatic polyester chain.

Once these bonds are broken, the fractions of the chains can migrate toward the reaction medium or can be incorporated into the crystal, due to the mobility afforded by the assay temperature $\left(37^{\circ} \mathrm{C}\right)$, since the PDO suture is a thermoplastic polymer composed by an amorphous and a crystalline phase, and the vitreous transition temperature $\left(\mathrm{Tg}-16^{\circ} \mathrm{C}\right)[12]$ is lower than the assay temperature. In this manner, when the migration of the chains toward the reaction medium or toward the crystal occurs, we can observe the empty spaces that are increasing in diameter, as seen in Figure $3 \mathrm{~b}$ 
(with possible loss of weight of the sample which could be detected by simple electronic weighing of the sample, before and after immersion).

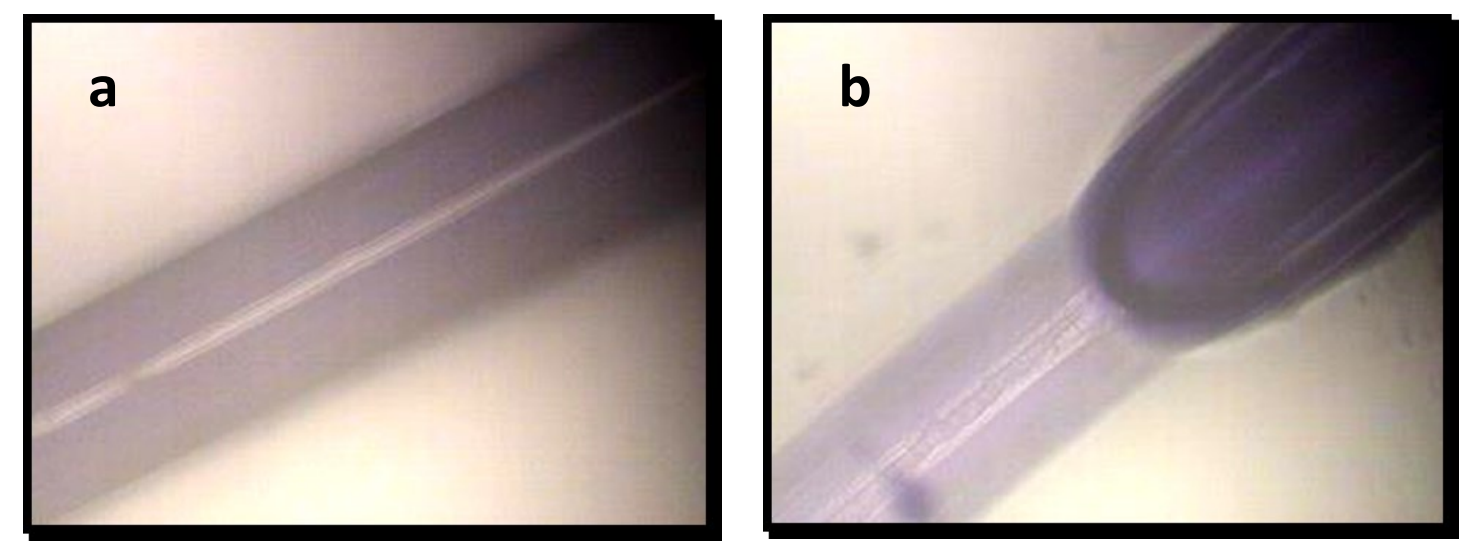

Figure 3 - PDO thread after 48 hours of immersion in HA.

a.Reflected light microphotography of the thread at $4 x$. Linear pattern of the hydrolytic attack with emphasis in the central column of the thread, which is seen as an empty band. b. Representative closeup of multiple regions of the thread that shows areas of central hydrolytic degradation with possible loss of mass of the polymer.

After 72 hours, with the release of the pigment we can observe empty spaces in the central column of the thread. Additionally, we can observe the presence of small superficial filaments on the periphery corresponding to the disorganization of the peripheral fibrils that show up as a frayed pattern along the fiber (Figure $4 \mathrm{a}$ and $\mathrm{b}$ ).

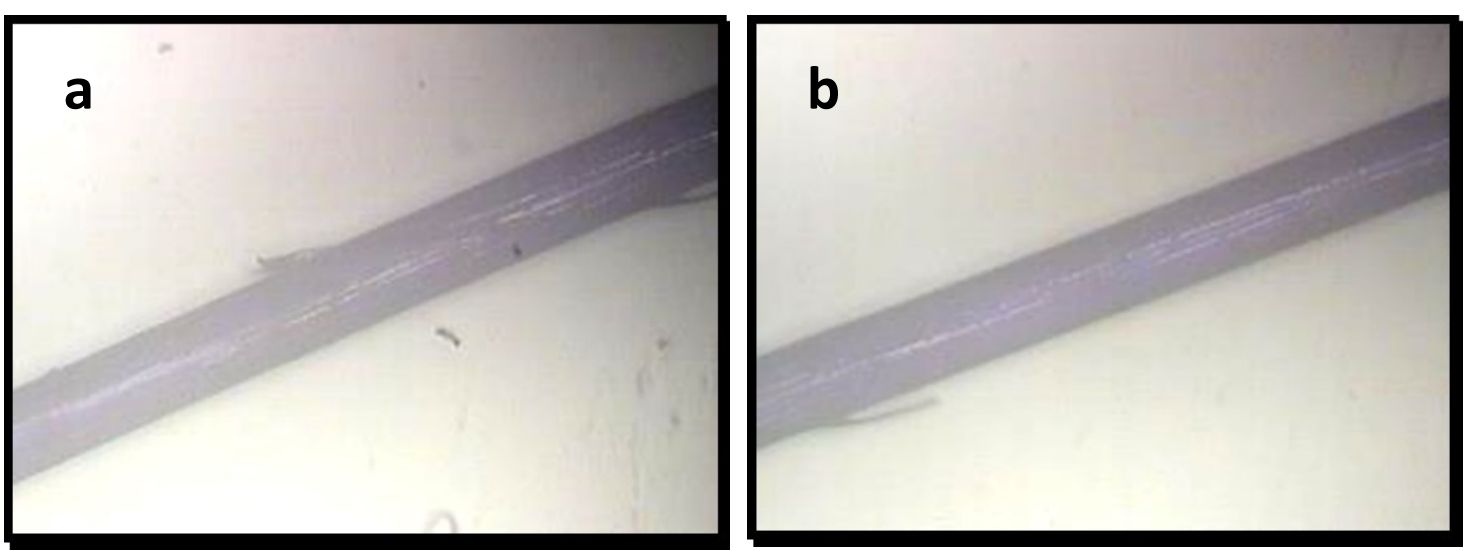

Figure 4-PDO thread after 72 hours of immersion in HA.

$a$ and $b$. Optical microphotography of the thread at $4 x$ showing a frayed pattern on the periphery of the thread.

In other areas of the segment submerged for 72 hours we observe the formation of small cracks, cross-wise to the suture thread, which indicates that water is diffusing into the fiber, with a tendency to accumulate during these first hours in the peripheral zone, which possibly corresponds to the amorphous zone of the material (Figure 5 a and b). 


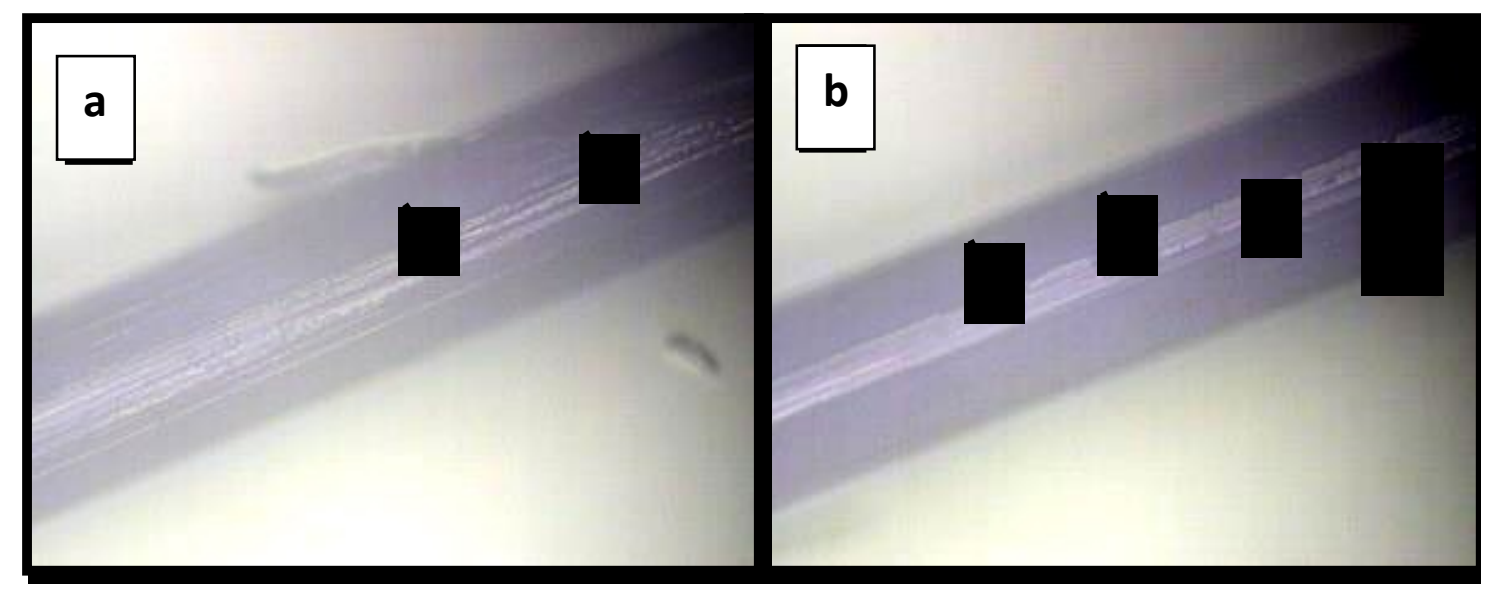

Figure 5 - PDO thread after 72 hours of immersion in HA.

$a$ and b. Optical microphotography of the thread at 10x. Arrows show transversal cracks due to migration of the amorphous zone to the crystalline zone of the polymer in the suture.

As shown in figure six, the propagation of the cracks from the center of the suture to the surface could corroborate that the degradation is heterogeneous and initially occurs on the surface of the samples and then, depending on the rate of water diffusion, penetrates toward the center of the thread.

Additionally, this could be related with the physical integrity of the suture during the first weeks, since it still has a central portion that has not been completely hydrolyzed. So as the central portion or skeleton becomes more exposed to the direct action of extracellular water, the process of degradation will become more acute, finally causing the suture to lose its stability as a fiber and the excision of the smaller reported fragments occurs, which can then be digested by the immune system.

\section{DISCUSSION}

Most of the known polymeric systems used in medicine are susceptible to degradation and the human physiological milieu has the appropriate conditions for easily producing hydrolytic processes. The most common biodegradative agents in the organism are the water on the extracellular matrix, salts (anions and cations), the $\mathrm{pH}$ of the physiologic medium (between 7 and 7.4) and enzymes. For this to occur, the polymer must possess hydrolytically unstable bonds, be hydrophilic so that the biodegradative process can occur in a reasonable amount of time, and the hydrolysis must be able to be carried out under physiologic $\mathrm{pH}$ conditions ${ }^{4}$. Based on our findings shown in the microphotographs depicting the behavior of the PDO threads through time, we consider 
that the PDO suture is a hydrophilic system with hydrolyzable bonds, causing the biodegradation time to be reduced in an aqueous medium such as $\mathrm{HA}$, to a level below the values estimated by the manufacturers.

The biodegradation is measured as the specific rate of absorption for each suture. This is the time in which a suture is degraded by the living organism and is expressed in days ${ }^{5}$. Resorption process by hydrolysis is caused by penetration of the fluids of the organism that break the molecules of the suture material. That is, water penetrates their structure causing them to dissolve. Thus, in this type of absorption there is a lower reaction of the organism ${ }^{5}$, which is desirable for facial esthetic and regenerative medical procedures. In face lifts, absorption of the PDO sutures is slow, it begins after 90 days and finalizes between 180 to 210 days, but the effect lasts for 2 to 3 years $^{13}$.

Moreover, all of the synthetic suture materials must show that a total loss of initial mass must require longer time that the loss of initial resistance. In the case of PDO, the rate of total loss of resistance occurs in about 63 day $^{13,14}$. However, the results observed in the microphotographs during our investigation show that the loss in mass of the PDO thread, when immersed in HA, is much faster, since at 72 hours, large areas of the thread already show empty interlaminar and interfibrillar spaces, suggesting loss of the matter forming the polymer.

The Food and Drug Administration of the United States approved the PDO thread system in 2005, and since then, it has been used for face lifts, dermal filling of deep folds, tensing secondary wrinkles, contouring of the facial oval minimizing the effects of sagging skin, and lipomatosis of the lower third of the neck. Among the most valued reasons for the use of PDO threads are their biologic properties, such as biocompatibility and resorption ${ }^{15}$.

Despite the aforementioned biological properties of PDO sutures and their use as scaffolds for active molecules in vivo ${ }^{15}$, associating hyaluronic acid to PDO tensor threads is an idea that has been spreading even though it is a practice that is not supported by any scientific evidence and is implausible from a biochemical standpoint, since addition of this molecule would promote a greater and faster degradation of the thread, as shown in the present investigation. 
All the information analyzed during our investigation led us to suggest the hypothesis that non-crosslinked hyaluronic acid is a powerful catalyst for the hydrolytic degradation of polydioxanone, since it is highly hydrophobic. That is, a chemical bond is established between the HA and the polymeric chains that make up the PDO suture. This is explained by the physicochemical characteristics of the PDO suture. It shows at least $0.5 \%$ water absorption, even at temperatures below $24^{\circ} \mathrm{C}$, and has poor resistance to diluted acids ${ }^{17}$. Thus, when associating the PDO with non-crosslinked HA, the chemical resistance of the biopolymer that makes up the suture is further reduced, possibly shortening the chains or opening the monomer bonds, giving rise to greater uptake of aqueous substances within the PDO structure. It is possible that the union of polydioxanone with hyaluronic acid results in a third species that is biochemically responsible for the final degradation of the suture, mimicking the role of lipase, the enzyme that classically degrades the hydrolyzed short chains of the PDO polymer ${ }^{17}$.

In addition, the constant uptake of water by HA molecules toward the suture allows for greater incorporation of ions capable of causing structural interlaminar and interfibrillar changes within the thread, until the strain stress module is reduced and deformed promoting failure or breakage of the thread. To understand this assumption, we must turn our attention away from the field of esthetic medicine and toward the discipline of biomaterials.

It is believed that these types of suture fibers are made up of fibrils that consist of a semicrystalline polymer (consisting of amorphous and crystalline regions) ${ }^{18}$. It is known that degradation of PDO occurs through hydrolysis, which results in the excision of the chain, mainly in the amorphous regions of the material ${ }^{19}$. This was confirmed in the present study in many of the microphotographs, where we repeatedly observed a pattern in which there were empty interfibrillar and interlaminar spaces over the axis of the fiber core.

From the microscopic point of view, the findings suggest that chain excision occurs during the first stage after hydration, beginning as soon as the chains are hydrated. Researchers report that the first change occurs after approximately 15 days 20 while in the present study it was observed much earlier, after 72 hours under assay conditions. 
It has been proposed that the changes at the level of the interfibrillar microstructure are the ones that finally cause rupture of the thread ${ }^{20}$. The results found during the present investigation coincide with those reported by these authors, since we observed indications of the beginning of excision of the chain as soon as the suture was hydrated with hyaluronic acid. Thus, as early as 24 hours, the microphotographs revealed qualitative changes in the physical structure of the fiber which become more evident at the microstructural interfibrillar level at 72 hours, as indicated by a series of empty spaces between the fibers, breakage of some peripheral fibers, and finally, loss of mass that is evident in the change in the diameter and weight of the suture.

Hyaluronic acid $(\mathrm{HA})$ is a polysaccharide belonging to the glycosaminoglycan family. It exists as a high molecular mass in synovial fluid that surrounds the joints, in cartilage and in the tissues of the eyes and skin. When hyaluronic acid is applied to wounds, water retention is improved ${ }^{20}$. Thus, if we consider the fact that HA favors water retention and also avoids fibrosis, adding HA to a highly hydrophilic PDO thread that is biodegradable through hydrolysis of its chains will definitely not prolong the expected results when implanting PDO threads in the dermis. Rather, it will induce relaxation of the stress or tensile strength of the suture by incorporating water into its interior, mitigate the sought after lifting effect expected from the fibrosis surrounding the suture, and dramatically reduce the permanence of the suture, a situation which counteracts the desired anchorage effect.

\section{CONCLUSION}

The non-crosslinked hyaluronic acid induces the rapid biodegradation of the PDO thread through hydrolysis, which begins after 24 hours of contact of the thread with the biomaterial. The hypothesis that we derive indicates that non-crosslinked hyaluronic acid is a powerful catalyst for the hydrolytic degradation of the PDO thread, since the latter is highly hydrophilic.

Thus, we propose that clinically, embedding PDO threads with HA will only accelerate biodegradation of the suture. For this reason, it could be that administrating HA could be indicated when facing complications for superficial placement of support threads, fulfilling the purpose of accelerating biodegradation of the thread.. 


\section{REFERENCES}

1. Sulamanidze MG, Sulamanidze I, Vozdvizhenskiy I, Sulamanidze K, Kadzhaya A. New method of face elastic thread lift. In: Clinic of Plastic, Aesthetic Surgery and Dermatocosmetology. Total Charm, 2013. DOI: 10.5772/56316v

2. Ali YH. 2 years outcome of thread lifting with absorbable barbed PDO threads: Innovative score for objective and subjective assessment. J Cosmet Laser Ther. 2018; 20(1):41-49. DOI: 10.1080/14764172.2017.1368562

3. Tejero Paloma. Hilos de sustentación facial: revisión de técnicas y materiales. SOARME NEWS. 8-18. Available in: http://www.academiamedicinaestetica.cl/assets/hilos-de-sustentacion-facial.pdf

4. Rifé Busquet, Mireia. Síntesis de copolímeros a partir de glicolida y p-dioxanona. Anexo II: Polímeros en suturas quirúrgicasPág. 1-50. Trabajo final de carrera. Universitat Politécnica de Catalunya, Barcelona-España 2007. Available in: https://upcommons.upc.edu/bitstream/handle/2099.1/3104/36143-

3.pdf?sequence $=3 \&$ isAllowed $=y$

5. Pestana-Tirado, R. A; Ariza-Solano, G. J; Palmett-Oviedo, D. M; González di-Filippo, A. Materiales de sutura: principios que determinan su escogencia. Rev. Colomb. Cir;14(4):253-262, 1999

6. Kim J, Kim HS, Seo JM, Nam KA, Chung KY. Evaluation of a novel thread-lift for the improvement of nasolabial folds and cheek laxity. J Eur Acad Dermatol Venereol. 2017; 31:e136-e179.

7. San Román, J. Polímeros biodegradables de interés en cirugía I: síntesis, propiedades y mecanismos biodegradativos. Revista de Plásticos Modernos 1990; 413:689-704.

8. Roby M, Kennedy J. Sutures (2004). Biomaterials Science - Introduction to materials in medicine, 2nd Edition, Elsevier Academic Press, p.614-627.

9. Ilankovan V. Recent Advances in Face Lift to Achieve Facial Balance. J Maxillofac Oral Surg. 2017; 16(1):3-12. doi: 10.1007/s12663-016-0981-8.

10. De las Heras Cristina. Técnicas antiptosis con hilos de suspensión subdérmica. Revista de la Asociación de Medicina Estética de Castilla- La Mancha (AMECLM) 2014; $4 . \quad$ Available in: http://ameclm.org/wpcontent/uploads/2012/10/revista_anual_AMECLM_4.pdf

11. De Masi E, De Masi F, De Masi R. Suspension Threads. Facial Plast Surg 2016;32:662-63. DOI http://dx.doi.org/10.1055/s-0036-1597541.

12. Zhang X. Physics properties of biodegradable polyester. Rev. MacromoL Cbem. Phy. 1993; C33(1): 81-102. 
13. Medeiros, AM; Araújo-Filho I; Carvalho, MDF. Fios de sutura. J Surg Cl Res. 2016; 7 (2):74-86

14. Sabino M, Márquez L y Feijoo J. Estudio de la degradación hidrolítica de la polidioxanona para la predicción del tiempo de vida útil Rev. Tec. Ing. Univ. Zulia. 1998; 21(3):170-178. Available in: http://www.produccioncientifica.luz.edu.ve/index.php/tecnica/article/viewFile/5 $608 / 5598$

15. Sarigul Guduk S, Karaca N. Safety and complications of absorbable threads made of poly-L-lactic acid and poly lactide/glycolide: Experience with 148 consecutive patients. J Cosmet Dermatol. 2018; 00:1-5.

16. 3rd Generation Aptos threads with HA for simultaneous lifting and rejuvenation. Aptos. global [Internet]. Tbilisi, Giorgia: APTOS International LTD; 2018 [citado 6 Ago 2018]. Available in: https://www.aptos.global/en/post/73/3rd-generationaptos-threads-with-ha-for-simultaneous-lifting-and-rejuvenation

17. George Wypych. PDO polydioxanone. In Handbook of Polymers (Second Edition). Canada. Chem Tec Publishing; 2016. Pages 346-348. ISBN: 9781927885116 Available in: https://doi.org/10.1016/C2015-0-01462-9

18. Peterlin A. Morphology and properties of crystalline polymers with fiber structure. Text Res J 1972;42:20.

19. C. Ping Ooi, R.E. Cameron The hydrolytic degradation of polydioxanone (PDOII) sutures. Part I: morphological aspects. J. Biomed. Mater. Res. 2002; 63 (3): 280290

20. Neuman M, Nanau RM, Oruña-Sanchez L, Coto G. Hyaluronic Acid and Wound Healing. J Pharm Pharm Sci (www.cspsCanada.org) 2015; 18(1): $53-60$ 